\title{
Simultaneous determination of oxygen and temperature using quantum dots and a ruthenium complex
}

\author{
P. A. S. Jorge ${ }^{* a}$, A. J. Silva ${ }^{\mathrm{b}}$, R. Benrashid ${ }^{\mathrm{c}}$, J. L. Santos ${ }^{\mathrm{ab}}$, F. Farahi ${ }^{\mathrm{c}}$ \\ ${ }^{\mathrm{a}}$ Unidade de Optoelectrónica e Sistemas Electrónicos. INESC Porto. \\ ${ }^{\mathrm{b}}$ Dept. de Física da Faculdade de Ciência da Universidade do Porto. \\ Rua do Campo Alegre, 687, 4169-007 Porto, Portugal; \\ ${ }^{\mathrm{c}}$ Department of Physics and Optical Science, University of North Carolina at Charlotte. \\ Charlotte, NC 28223, USA.
}

\begin{abstract}
An all-optical scheme for simultaneous determination of oxygen and temperature is presented. A ruthenium complex immobilized in a non-hydrolytic sol-gel matrix is used as oxygen sensor. Temperature information is provided by CdSe quantum dots immobilized in the same material. While the dye luminescence is quenched by oxygen and temperature, the nanocrystals luminescence depends only on temperature. Results presented demonstrate that the combined luminescence response allows to simultaneously assess both parameters using a single optical fiber system.
\end{abstract}

Keywords: Quantum-dots; luminescence; optical oxygen sensors; temperature

\section{INTRODUCTION}

Luminescence based biochemical sensing allows fast and sensitive detection. Recent technological advances in different areas have concurred for the feasibility of luminescence spectroscopy in the tip of an optical fiber[1]. In this context, analytical tools for remote and real time detection, with minimum intrusion, can be fabricated. Presently, biochemical optical fibers sensors can be found as commercial products (eg. $\left.\mathrm{O}_{2}, \mathrm{pH}\right)$. However, in spite of great advances, some limitations still arise. Luminescence mechanisms are temperature dependent and, therefore, the correct determination of the analyte is only possible at known temperature. Schemes for simultaneous determination of temperature and a chemical parameter have been proposed. Liao et al. demonstrated the use of an alexandrite crystal together with a Platinum complex to detect oxygen and temperature with the same fiber probe [2]. Recently, Wolfbeis et al. synthesized a composite luminescent material allowing dual sensing of oxygen and temperature by lifetime methods[3]. Nevertheless, commercial sensors still rely on conventional technologies for temperature measurement and all-optical solutions for simultaneous detection of temperature and a biochemical parameter are highly desirable.

Quantum dots (QD), are nanometer sized particles of semiconductor material with potential to solve many problems of luminescence sensors[4]. When compared with traditional dyes, they show narrow emission spectrum, a broad absorption band, high quantum yields, increased photostability and the ability to tune their properties by changing the nanocrystals size or composition. Available in a wide range of wavelengths they have a great potential for multiplexing applications. QD have recently been reported as suitable luminescent temperature probes[5]. In this work we propose the combination of QD with an oxygen sensitive dye to obtain a sensor whose spectral characteristics allows simultaneous measurement of oxygen and temperature. Results are presented demonstrating the feasibility of this concept.

\section{PROPOSED CONFIGURATION}

\subsection{Principles of operation}

The dynamic quenching of luminescence is the most widely used oxygen sensing mechanism. When excited by blue LEDs, organometallic ruthenium complexes present luminescence emission which is strongly quenched by oxygen. Their immobilization in sol-gel hosts is straightforward, making them suitable for optical fiber applications [6]. The behavior of both the luminescence intensity, I, and the excited state lifetime, $\tau$, of these sensing dyes in the presence of oxygen can be described by the Stern-Volmer (SV) equation[7]:

\footnotetext{
**pjorge@inescporto.pt; phone +351 226082 601; fax +351 226082 799; www.inescporto.pt
} 


$$
\frac{I_{0}}{I}=\frac{\tau_{0}}{\tau}=1+K_{S V}\left[O_{2}\right]
$$

where $\mathrm{I}_{0}$ and $\tau_{0}$ are the unquenched luminescence intensity and lifetime, respectively, $\mathrm{K}_{\mathrm{SV}}$ is the $\mathrm{SV}$ constant, and [ $\left.\mathrm{O}_{2}\right]$ is the oxygen concentration. This equation establishes a linear relation between $\mathrm{I}_{0} / \mathrm{I}$ and $\left[\mathrm{O}_{2}\right]$, that can be used for calibration purposes. When the sensing dye is immobilized in a solid host, however, heterogeneous distribution within the host can lead to deviations from this behavior. Such situations must be described by a two site-model[7].

Temperature has a dual effect on the sensor calibration function. Increasing temperature introduces higher probability of non-radiative transitions, thus decreasing the luminescence yield and the excited state lifetime. In the room temperature range, this translates in a linear dependence of luminescence intensity with temperature. In addition, because $\mathrm{K}_{\mathrm{SV}}$ is proportional to the diffusion coefficient of oxygen into the sensing membrane, it is also temperature dependent. Typically, increased temperature results in higher quenching efficiency and, therefore, higher values of $\mathrm{K}_{\mathrm{Sv}}$. This way, an independent temperature measurement is needed, in order to univocally retrieve the oxygen concentration.

QD can be used to provide such independent measurement. Core shell QD immobilized in a non-hydrolytic sol-gel material are resistant to oxidation, presenting an oxygen independent luminescence output. Conversely, it has been shown that both the luminescence intensity and the peak emission wavelength of QD, have a linear and reversible dependence on temperature in the environmental range. When temperature is increased, luminescence intensity decreases, due to increase of non-radiative transitions, and the peak wavelength is shifted towards longer wavelengths, due to expansion of the crystalline lattice inducing a decrease in the material bandgap energy. Either effect can be used to obtain temperature information. However, simple intensity measurements are prone to error due to optical power fluctuations. In a previous work we have demonstrated a detection scheme allowing self-referenced temperature measurements with QD[8]. It involves detection of two signals, A and B, corresponding to two narrow spectral windows on opposite sides of the emission spectrum, and subsequent processing according to $S_{Q D}=(A-B) /(A+B)$. Due to the presence of a wavelength shift $\mathrm{S}_{\mathrm{QD}}$ is proportional to temperature and independent of the system optical power level.

\subsection{Experimental setup}

CdSe-ZnS core-shell nanocrystals, purchased from Nanoco, and [Ru(dpp)]-Tris(4,7-diphenyl-1,10-phenanthrolin) (I)chloride hexahydrate, purchased from Sigma, were immobilized in a non-hydrolytic sol-gel material. QD with peak emission around $520 \mathrm{~nm}$ were chosen to avoid overlap with $\mathrm{Ru}(\mathrm{dpp})$ luminescence (595 nm).Thin films and bulk samples with different thicknesses were made by spin coating or casting. Details of sol-gel preparation and coating procedures are described elsewhere[8,9]. To demonstrate the feasibility of the proposed concept, oxygen sensing membranes doped with Ru(dpp) and reference membranes doped with QD were interrogated in the setup of figure 1.

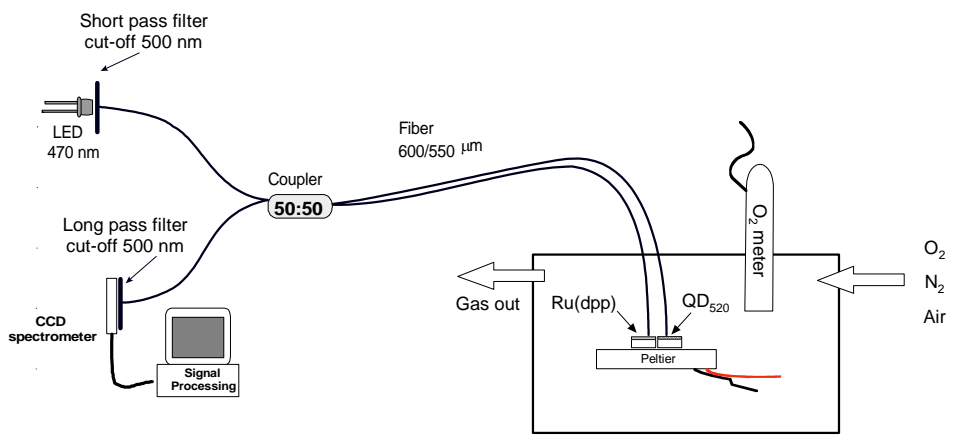

Figure 1 Experimental setup used for simultaneous determination of oxygen and temperature.

An optical fiber coupler (50/50 coupling ratio; core/cladding: silica/silica, 550/600 $\mu \mathrm{m}$ ) was used to perform excitation and collection of luminescence. A blue LED, followed by a short pass filter (cutoff $500 \mathrm{~nm}$ ), was used as excitation source. The sensing samples were placed on top of a peltier cooling device, which allowed to change their temperature, inside a gas chamber with controlled atmosphere. Collection of luminescence was performed by the distal ends of the coupler outputs. Detection was then made using a CCD spectrometer with additional filtering (long pass, cutoff $500 \mathrm{~nm}$ ). Dedicated software allowed to implement signal processing on the detected spectral outputs. The setup was used to submit the samples to controlled changes of temperature and oxygen concentration. After sensor calibration, and some signal processing, it was possible to perform simultaneous measurement of both parameters. 


\section{RESULTS AND DISCUSSION}

In order to obtain the sensor calibration curves, the two samples were subjected to controlled and alternate changes of oxygen concentration and temperature. In figure 2 the spectral outputs of the combined response can be observed. Figure 2a shows the behavior of QD and Ru(dpp), at a constant temperature, when subjected to different atmospheres (oxygen content ranging from $0 \%$ to $100 \%$ ). Conversely, in figure $2 \mathrm{~b}$, it is shown the behavior of the same samples, in a $20.9 \%$ oxygen atmosphere, when subjected to temperatures ranging from $16^{\circ} \mathrm{C}$ to $40^{\circ} \mathrm{C}$.
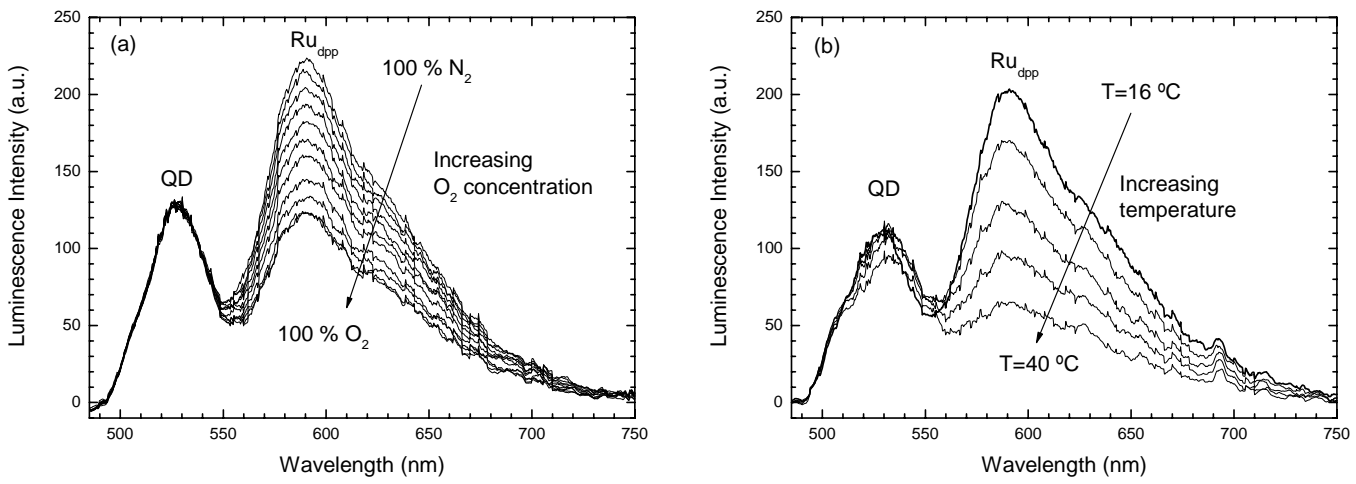

Figure 2 Spectral response of sensing films subjected to: (a) changes of oxygen concentration; (b) temperature changes.

It can be observed that while oxygen strongly quenches the luminescence of Ru(dpp), it has little or no effect on the QD luminescence. This was the case when the relative luminescence intensities of Ru(dpp) and QD were similar. However, in situations where $\mathrm{Ru}(\mathrm{dpp})$ had a much stronger signal, the small spectral overlap originated crosstalk. In the present scheme, this situation was avoided by balancing the luminescent output of both samples, controlling film thickness and doping concentrations. Temperature, on the other hand, strongly affects both luminescence signals. Both samples are strongly quenched, with a wavelength shift being observed in the QD emission. From these results, the sensor calibration curves could be obtained (figure $3 \mathrm{a}$ and $3 \mathrm{~b}$ ).
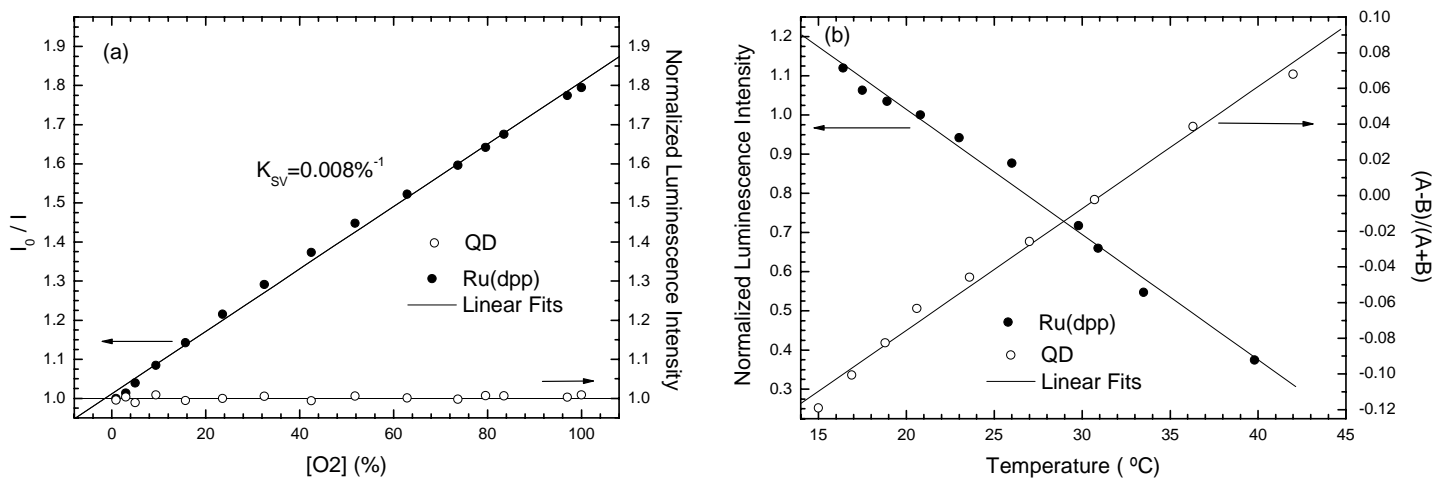

Figure 3 Sensor calibration curves. (a) Response to oxygen concentration; (b) Response to temperature changes.

The obtained SV plot is slightly non-linear. Nevertheless, a reasonable linear fit $(\mathrm{R}=0.997)$ could be obtained, which was used as an approximation $\left(\mathrm{K}_{\mathrm{SV}}=0.008 \%{ }^{-1}\right)$. Both the luminescence intensity and peak wavelength of the QD have shown to be insensitive to $\mathrm{O}_{2}$ concentration; fluctuations observed over the full concentration range were smaller than $0.6 \%$ for the intensity response, and smaller than $3 \%$ for the wavelength response $\left(\mathrm{S}_{\mathrm{QD}}\right)$. These residual fluctuations, are partly due to a small thermal drift, which took place during the tests. Both samples showed a linear dependence on temperature.

By using the calibration data, a simple test was made to assess the capability of the system for simultaneous measurement. The sensing samples were subjected to successive cycles of air and nitrogen. Initially this was done at a slightly elevated temperature (around $24^{\circ} \mathrm{C}$ ), at a certain point $(\mathrm{t}=360 \mathrm{~s})$, the temperature was lowered to room values (around $20^{\circ} \mathrm{C}$ ). The resulting measurements can be seen in figure 4. Figure 4a shows the oxygen concentration as measured by the luminescence of Ru(dpp). While at ambient temperature accurate oxygen concentrations of $20.9 \%$ (air) 
and $0 \%\left(\mathrm{~N}_{2}\right)$ were measured, at a higher temperature, the retrieved oxygen concentrations are of $30 \%$ (air) and $10 \%\left(\mathrm{~N}_{2}\right)$ showing the deleterious effect of temperature. Figure $4 \mathrm{~b}$ shows the temperature information recovered by the QD processed signal, clearly showing the applied temperature step and some thermal drift of the peltier. In order to obtain an accurate oxygen measurement, this information was then used to correct the measured luminescence intensity of Ru(dpp) for the effect of temperature. Introducing the corrected intensity value in the SV calibration function, an accurate oxygen measurement could then be obtained in the whole temperature range (figure 4c).

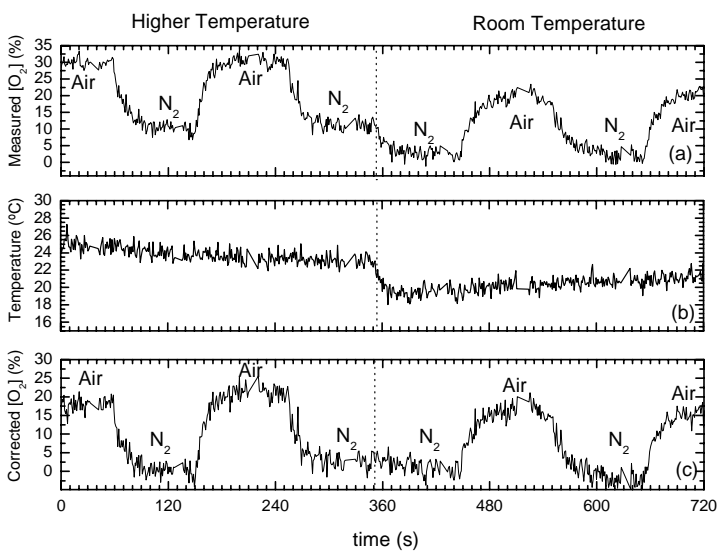

Figure 4 Sensor output when subjected to simultaneous change of oxygen and temperature: (a) $\mathrm{O}_{2}$ measured by Ru(dpp);

(b) temperature measured by QD; (b) temperature compensated $\mathrm{O}_{2}$ measurement.

Because a small temperature step was applied, for simplicity the effect of temperature in the value of $K_{S v}$ was not accounted for. Due to this approximation, and to the non-homogeneous heating of the test chamber, a small temperature induced drift can still be observed in the corrected signal. Nevertheless, full characterization of $\mathrm{K}_{\mathrm{Sv}}(\mathrm{T})$, together with optimization of the temperature control system will surely allow for temperature independent oxygen sensing in a broad temperature range.

\section{CONCLUSION}

An all-optical scheme for simultaneous determination of oxygen and temperature was proposed and demonstrated. Results obtained showed the feasibility of using QD in combination with a ruthenium dye, both immobilized in a sol-gel host, to design dual sensing membranes. Due to the versatility of QD and the sol-gel process, the demonstrated principles can be applied to any luminescence based sensor. Coating a single fiber with these sensing membranes, will allow implementation of miniaturized multiparameter sensing heads with great potential for medical and environmental use.

\section{REFERENCES}

1. Wolfbeis, O.S., Fiber-optic chemical sensors and biosensors. Analytical Chemistry, 2006. 78(12): p. 3859-3873.

2. Shih-Chu Liao, et al., Real-Time Frequency Domain Temperature and Oxygen Sensor with a Single Optical Fiber. IEEE Transact. on Biomedical Eng., 1997. 44(11).

3. Borisov, S.M., et al., Composite luminescent material for dual sensing of oxygen and temperature. Advanced Functional Materials, 2006. 16(12): p. 1536-1542.

4. Catherine J. Murphy, Optical sensing with quantum dots. Analytical Chemistry, 2002. 74: p. 520A-526A.

5. Glen W. Walker, et al., Quantum-dot optical temperature probes. Appl. Physics Lett., 2003. 83(17): p. 3555-3557.

6. Aisling K. McEvoy et al.. Dissolved oxygen sensor based on fluorescence quenching of oxygen-sensitive ruthenium complexes immobilized in sol-gel-derived porous silica coatings. Analyst, 1996. 121: p. 785-788.

7. Joseph R. Lakowicz, Principles of Fluorescence Spectroscopy. 2 ed. 1999, New York: Kluwer-Plenum.

8. Jorge, P.A.S., et al., Quantum dots as self-referenced optical fibre temperature probes for luminescent chemical sensors. Measurement Science \& Technology, 2006. 17(5): p. 1032-1038.

9. Ramazan Benrashid and P. Velasco, High performance sol-gel spin-on glass materials, U.S. patent 0022697 A1 (3 February 2005). 